\title{
ORIGINAL
}

\section{ESTILO DE VIDA Y ADHERENCIA AL TRATAMIENTO DE LA POBLACIÓN CANARIA CON DIABETES MELLITUS TIPO 2}

\author{
Antonio Cabrera de León (1,2), José Carlos del Castillo Rodríguez (1), Santiago Domínguez \\ Coello (1), María del Cristo Rodríguez Pérez (1), Buenaventura Brito Díaz (1), Carlos Borges \\ Álamo (1), Lourdes Carrillo Fernández (1), Delia Almeida González (1), José Juan Alemán \\ Sánchez (1), Ana González Hernández (1) y Armando Aguirre-Jaime (1). \\ (1) Unidad de Investigación de Atención Primaria y del Hospital Universitario Nuestra Señora de La Candelaria. Tenerife. \\ (2) Universidad de La Laguna. Tenerife.
}

\section{RESUMEN}

Fundamento: la población canaria sufre la mayor mortalidad por diabetes tipo 2 (DM2) en España. El objetivo de este estudio es conocer el estilo de vida de las personas diabéticas del archipiélago y su adherencia al tratamiento, así como la DM2 desconocida.

Métodos: estudio transversal de 6.729 personas de la población general participantes en la cohorte "CDC de Canarias" (edad 18-75 años). Se obtuvieron sus antecedentes médicos, dieta, actividad física, medicamentos consumidos, tabaco, etc.

Resultados: la prevalencia de DM2 fue $12 \%$ en varones y $10 \%$ en mujeres $(\mathrm{p}=0,005)$. El $22 \%$ de varones y $9 \%$ de mujeres desconocían su enfermedad $(\mathrm{p}<0,001)$. Sumando DM2 desconocida, DM2 no tratada e incumplimiento terapéutico, el $48 \%$ de los hombres y $28 \%$ en mujeres no seguían tratamiento correcto. Los varones diabéticos eran más obesos que los no diabéticos (45 versus $25 \%$; $\mathrm{p}<0,001$ ) pero no presentaban diferencias en tabaquismo ( $\left.28 \% ; \mathrm{IC}_{95 \%}=23-33\right)$ o sedentarismo $\left(62 \% ; \mathrm{IC}_{95 \%}=56-68\right)$. Las mujeres diabéticas también eran más obesas $(54$ versus $27 \%$; $\mathrm{p}<0,001) \mathrm{y}$, aunque fumaban menos (11 versus $22 \%$; $\mathrm{p}<0,001)$, eran igual de sedentarias $(75 \%$; $\left.\mathrm{IC}_{95 \%}=70-79\right)$. La ingesta calórica era menor en quienes sufrían DM2 ( $<<0,001)$, pero el $93 \%\left(\mathrm{IC}_{95 \%}=91-95\right)$ superaba el consumo recomendado de grasas saturadas y el $69 \%$ (IC95\%=65-72) padecía síndrome metabólico.

Conclusiones: la población diabética en Canarias es sedentaria y obesa, muestra un consumo alto de grasas saturadas y gran prevalencia de síndrome metabólico. El porcentaje que sigue tratamiento regular es muy bajo, sobretodo en varones, que además mantienen el tabaquismo.

Palabras clave: Diabetes mellitus. Adherencia al tratamiento. Estilo de vida. Tabaquismo. Obesidad.

Correspondencia:

Antonio Cabrera de León

Unidad de Investigación.

Hospital Universitario NS de Candelaria

Carretera de El Rosario 145.

38010 Santa Cruz de Tenerife.

Correo electrónico: acableo@gobiernodecanarias.org
ABSTRACT

\section{Lifestyle and Treatment Adherence of Type 2 Diabetes Mellitus People in the Canary Islands}

Background: The Canary Islands population experiences the highest type 2 diabetes (DM2) mortality in Spain. We studied lifestyle, unknown DM2 and treatment adherence in diabetics of these islands.

Methods: cross-sectional study of 6729 subjects from the general population (age 18-75) that participate in the cohort study "CDC of the Canary Islands". We found out their medical problems, diet, physical activity, medications, smoking, etc.

Results: Prevalence of DM2 was $12 \%$ in men and $10 \%$ in women $(p=0.005)$. The disease was unknown in $22 \%$ of men and $9 \%$ of women $(\mathrm{p}<0.001)$. Considering unknown DM2, lack of treatment and lack of adherence, $48 \%$ of men and $28 \%$ of women did not follow a regular treatment. Diabetics' men prevalences of smoking (28\%; $\left.\mathrm{CI}_{95 \%}=23-33\right)$ and sedentariness $\left(62 \% ; \mathrm{CI}_{95 \%}=56-68\right)$ were similar to non diabetic men, but obesity was more frequent in diabetics ( 45 versus $25 \%$; $\mathrm{p}<0.001$ ). Diabetics women showed a higher obesity (54 versus $27 \%$; $\mathrm{p}<0.001)$ and a lower smoking prevalence (11 versus $22 \%$; $\mathrm{p}<0,001)$ than non diabetics, but they presented a similar sedentariness $\left(75 \% ; \mathrm{CI}_{95 \%}=70-79\right)$. In both sexes, energy intake was lower in diabetics $(\mathrm{p}<0.001)$, but $93 \%$ of them $\left(\mathrm{CI}_{95 \%}=91-95\right)$ showed a high consumption of calories from saturated fat and $69 \%\left(\mathrm{CI}_{95 \%}=65-72\right)$ presented metabolic syndrome.

Conclusions: The Canarian diabetics are a sedentary and obese population that show a high consumption of saturated fats and high prevalence of metabolic syndrome. The proportion of them following regular treatment is low, specially in diabetic men that, in addition, still smoke.

Keywords: Diabetes Mellitus. Medication Adherence. Life Style. Smoking. Obesity.

Financiación:

Fondo de Investigaciones Sanitarias (PI 070934). 


\section{INTRODUCCIÓN}

La diabetes mellitus tipo 2 (DM2) es un problema de salud importante en las Islas Canarias puesto que su población sufre la mayor mortalidad de España por esta enfermedad y la diferencia con el resto del país es grande. En 2005 los hombres de esta Comunidad presentaron una mortalidad $\left(32,97 / 10^{5}\right) 4$ veces mayor que la de sus congéneres de Madrid $\left(8,44 / 10^{5}\right)$ y 3 veces superior a la del conjunto de España $\left(10,42 / 10^{5}\right)$. En el mismo año, las mujeres diabéticas de Canarias $\left(23,07 / 10^{5}\right)$ multiplicaron por más de 3 la tasa de la Comunidad de Madrid $\left(6,93 / 10^{5}\right)^{1}$. En 2006 la mortalidad total por DM2 en Canarias $\left(30,70 / 10^{5}\right)$ fue casi 5 veces superior a la de Madrid $\left(6,40 / 10^{5}\right)$, y si se analiza la mortalidad prematura por DM2, es decir la que ocurre antes de los 75 años de edad, entonces la tasa del archipiélago $\left(10,8 / 10^{5}\right)$ casi multiplicó por 6 a la de Madrid $\left(1,9 / 10^{5}\right)^{2}$. Aunque la metodología pueda no ser uniforme en todas las Comunidades al recoger los datos de mortalidad, estas diferencias son demasiado grandes para atribuirlas a ello en un sistema sanitario tan homogéneo como el español.

Por otra parte, cuando se ha estimado el riesgo cardiovascular de las personas diabéticas de Canarias se ha comprobado que casi dobla al de Gerona ${ }^{3}$. Y además, en estas islas se da también la mayor incidencia $\left(187 / 10^{6}\right)$ de España en insuficiencia renal crónica dependiente de tratamiento renal sustitutivo (diálisis y trasplante), de la cual es la diabetes la causa conocida más frecuente $^{4}$ y aparece asociada al $40 \%$ de los trasplantes renales ${ }^{5}$. Probablemente no sea ajeno a todo lo anterior el que esta Comunidad encabece también la mortalidad nacional por cardiopatía isquémica $\left(164,81 / 10^{5}\right.$ para los hombres y $78^{\prime} 96 / 10^{5}$ para las mujeres de la provincia de Las Palmas en el año 2000) ${ }^{6}$.

La población del archipiélago está hoy tipificada antropológica, histórica y genéti- camente: étnicamente caucasiana, culturalmente española y europea y genéticamente situada en el conjunto de las poblaciones ibéricas $^{7}$, formada principalmente por el mestizaje entre los españoles que colonizaron las islas y los aborígenes de procedencia norteafricana que en ellas habitaban ${ }^{8}$. Eso explica que los canarios sean la población de España que presenta menor distancia genética con las poblaciones norteafricanas, como demostración de la supervivencia genética de lo aborigen en el $70 \%$ de sus habitantes ${ }^{7}$. No obstante, no existe ningún determinante genético conocido que explique la elevada prevalencia de DM2 en Canarias (11-13\% para la población general adulta estandarizada por edad $^{3,9,10}$ ), ni mucho menos la pésima evolución clínica que los datos arriba expuestos sugieren. Por el contrario, la prevalencia puede ser en parte explicada por la actual epidemia de obesidad, de la cual estas islas son un buen ejemplo pues presentan en su población adulta las mayores prevalencias de obesidad $(30 \%)$ y sobrepeso $(39 \%)$ descritas en el país ${ }^{3}$. Pero el problema principal no es sólo la alta prevalencia de DM2 de esta enfermedad en Canarias sino la mala evolución de la enfermedad. Estudios basados en los registros de pacientes con DM2 en la atención primaria de esta Comunidad indican que la frecuencia de tratamiento con fármacos es alta ${ }^{11}$. A su vez, estudios hospitalarios han comprobado que las condiciones en que se remiten los pacientes diabéticos a las consultas especializadas son similares a las de otras regiones ${ }^{12}$. Parece, entonces, poco probable que exista un abordaje clínico-terapéutico de los pacientes diabéticos sustancialmente peor que el de otras latitudes, y se hace necesario plantear otras hipótesis.

El objetivo de este estudio es averiguar si en la población diabética de Canarias existe un estilo de vida inadecuado para la enfermedad, una baja adherencia al tratamiento o una elevada prevalencia de DM2 desconocida. 


\section{SUJETOS Y MÉTODOS}

Se realizó un estudio transversal de los sujetos participantes en la cohorte $« \mathrm{CDC}$ de Canarias», que habían sido seleccionados aleatoriamente de la población general entre los años 2000 y 2005 (CDC son las siglas de Cardiovascular, Diabetes, Cáncer). La metodología seguida en el reclutamiento ha sido descrita en un trabajo anterior $^{13}$. Brevemente diremos que ésta es la mayor muestra de población general estudiada hasta el momento en el archipiélago (edad: 18-75 años). La tasa de participación alcanzó el $70 \%$, siendo la muestra finalmente reclutada de 6.729 individuos. A cada participante se le entrevistó en persona empleando un cuestionario sobre sus antecedentes médicos personales y familiares y su estilo de vida: dieta, actividad física, trabajo, consumo de medicamentos, consumo de tabaco, alcohol, etc (cuestionario CDC, disponible en: www.icic.es/cuestionarioCDC/docs). También se les efectuó una exploración física para conocer su antropometría y constantes vitales, además de una extracción de sangre venosa en ayunas para determinaciones bioquímicas. Este estudio fue aprobado por el Comité de Bioética del Hospital Universitario Nuestra Señora de la Candelaria y todos los participantes firmaron su consentimiento informado.

Siguiendo los criterios de la $\mathrm{ADA}^{14}$ se aceptó como diabética a toda persona que manifestara serlo y estuviera en tratamiento farmacológico-dietético prescrito por su médico, o a quien sin saber que era diabético tuviera una glucemia en ayunas mayor de $125 \mathrm{mg} / \mathrm{dl}$. En este último caso se realizó una segunda determinación de glucemia basal para confirmar el diagnóstico. Se definió el sedentarismo como no realizar al menos 30 minutos diarios de actividad física modera$\mathrm{da}^{15} \mathrm{y}$ la obesidad como padecer un índice de masa corporal $(\mathrm{IMC}=$ peso en $\mathrm{Kg} /$ talla en $\mathrm{m}^{2}$ ) superior a 30. Como definición del síndrome metabólico se utilizó la del ATPIII ${ }^{16}$. Se consideró fumador a quien declaró serlo.
Para ajustar los resultados por el nivel socioeconómico de los participantes, se empleó el índice REI de clase social, que había sido validado anteriormente en esta misma población ${ }^{17} \mathrm{y}$ mide la misma en función del nivel de estudios alcanzado más los ingresos familiares per cápita y el grado de hacinamiento en el hogar. La ingesta de calorías y nutrientes se obtuvo a través de un cuestionario de frecuencia y cantidad de consumo de alimentos que ha sido valida$\mathrm{do}^{18}$ y forma parte del cuestionario CDC arriba citado.

Las muestras sanguíneas fueron centrifugadas a temperatura ambiente a $2.000 \mathrm{rpm}$ durante 10 minutos, colocadas en hielo dentro de contenedores portátiles y trasladadas diariamente hasta el laboratorio del hospital. La glucemia y lipoproteínas fueron medidas con el autoanalizador Hitachi ${ }^{\circledR}$ 917 dentro de las primeras 24 horas desde la extracción y se expresaron en $\mathrm{mg} / \mathrm{dL}$. El colesterol LDL fue estimado mediante la diferencia: (colesterol total - HDL colesterol - triglicéridos/5).

Análisis estadístico: Las variables de escala se resumieron mediante su media $+/$ DE y las categóricas se presentaron como frecuencias relativas con sus intervalos de confianza al $95 \%\left(\mathrm{IC}_{95 \%}\right)$. La comparación de proporciones se efectuó con la prueba de la Chi cuadrado de Pearson y la de medias con una prueba de la t. Para ajustar por la edad y clase social la asociación de la DM2 con el tabaquismo, el sedentarismo y la obesidad, fueron empleados modelos de regresión logística, obteniendo las razones de ventaja con su intervalo al 95\% (OR; $\left.\mathrm{IC}_{95 \%}\right)$. Para estudiar la asociación entre DM2 e ingesta calórica ajustando por la edad y clase social se empleó un modelo de regresión lineal; en este caso los resultados se presentan como coeficiente de regresión con su error estándar ( $\pm \mathrm{EE})$. Los cálculos de $\mathrm{IC}_{95 \%}$ para las proporciones se efectuaron manualmente. El resto de los cálculos se realizó con el paquete estadístico SPSS $^{\odot}$ versión 15. 


\section{RESULTADOS}

La prevalencia de DM2 fue $12 \%$ en varones y $10 \%$ en mujeres (tabla $1 ; \mathrm{p}=0,005$ ). El $15 \%$ de las personas diabéticas desconocían su enfermedad, siendo mayor el desconocimiento en varones (tabla $1 ; \mathrm{p}<0,001$ ). Las diferencias entre sexos en cuanto a tratamiento de la diabetes no fueron significativas salvo en las recomendaciones dietéticas, que habían sido realizadas con menor frecuencia en los hombres (tabla 1; $\mathrm{p}<0,012$ ).

No hubo diferencias entre las mujeres diabéticas que conocían y las que desconocían padecer la enfermedad en lo que se refiere a edad, IMC, prevalencia de tabaquismo o de sedentarismo, pero la ingesta calórica fue menor en $100 \mathrm{Kcal} /$ día entre aquellas con DM2 conocida $(p=0,003)$. En los hombres diabéticos no se halló ninguna diferencia en estas variables por el hecho de que conocieran o no su padecimiento.

Sumando la diabetes desconocida a la no tratada, el 23\% de las mujeres y el 39\% de los hombres con DM2 no estaban en tratamiento regular. A lo cual habría que añadir el $5 \%$ de mujeres y $9 \%$ de varones que declararon cumplir inadecuadamente con el tratamiento. Esta diferencia fue significativa (48\% no tratados regularmente en los varones versus $28 \%$ en las mujeres; $\mathrm{p}=0,004)$.

A partir de este punto el resto de los resultados se presentan sólo para las personas que conocían su diabetes. La edad fue significativamente mayor en los participantes diabéticos (tabla 2), no se encontró dife-

Tabla 1

Prevalencia de diabetes tipo 2, conocimiento de la misma y tratamiento recibido por sexo. Los resultados son porcentajes $\left(\mathrm{IC}_{95 \%}\right)$

\begin{tabular}{|c|c|c|c|}
\hline & $\begin{array}{c}\text { Hombres } \\
(\mathrm{n}=2913)\end{array}$ & $\begin{array}{c}\text { Mujeres } \\
(\mathrm{n}=3816)\end{array}$ & $\mathrm{p}$ \\
\hline Prevalencia de diabetes tipo 2 & $11,8(10,6-13,0)$ & $9,7(8,8-10,6)$ & 0,005 \\
\hline Prevalencia de diabetes conocida & $9,2(8,2-10,2)$ & $8,8(7,9-9,7)$ & 0,542 \\
\hline Proporción de diabetes desconocida & $22,0(17,6-26,4)$ & $9,1(6,2-12,0)$ & $<0,001$ \\
\hline Proporción de diabetes conocida y tratada & $82,8(78,3-87,3)$ & $85,6(81,6-89,6)$ & 0,371 \\
\hline Proporción que recibe tratamiento dietético* & $70,3(64,8-75,8)$ & $79,8(75,5-84,1)$ & 0,012 \\
\hline Proporción que recibe tratamiento con ADO* & $60,9(55,1-66,7)$ & $52,7(47,4-58,0)$ & 0,081 \\
\hline Proporción que recibe tratamiento con insulina* & $16,5(12,1-20,1)$ & $20,5(16,2-24,8)$ & 0,424 \\
\hline Proporción que cumple el tratamiento con ADO a diario & $91,0(86,2-95,8)$ & $95,0(91,7-98,3)$ & 0,078 \\
\hline
\end{tabular}

* En cada persona pueden coexistir más de un tipo de tratamiento.

Tabla 2

Distribución de tabaquismo, sedentarismo, ingesta energética, IMC y obesidad en personas diabéticas y no diabéticas, estratificando por sexo

\begin{tabular}{|l|c|c|c|c|c|c|}
\hline \multirow{2}{*}{} & \multicolumn{3}{|c|}{ Hombres } & \multicolumn{2}{c|}{ Mujeres } \\
\cline { 2 - 7 } & $\begin{array}{c}\text { Diabetes } \\
(\mathbf{n = 2 6 9 )}\end{array}$ & $\begin{array}{c}\text { No diabetes } \\
(\mathbf{2 5 6 9 )}\end{array}$ & $\mathbf{p}$ & $\begin{array}{c}\text { Diabetes } \\
(\mathbf{n}=\mathbf{3 3 6})\end{array}$ & $\begin{array}{c}\text { No diabetes } \\
(\mathbf{3 . 4 4 7})\end{array}$ & $\begin{array}{c}\text { p } \\
\text { p }\end{array}$ \\
\hline Edad (años)* & $53,4 \pm 11,0$ & $42,0 \pm 12,5$ & $<0,001$ & $53,5 \pm 10,4$ & $42,0 \pm 12,6$ & $<0,001$ \\
\hline Tabaquismo** & $28,0(22,6-33,4)$ & $31,5(29,7-33,3)$ & 0,232 & $10,7(7,4-14,0)$ & $22,4(21,0-23,8)$ & $<0,001$ \\
\hline Sedentarismo** & $61,7(55,9-67,5)$ & $54,4(52,5-56,3)$ & 0,022 & $74,7(70,1-79,3)$ & $70,9(69,4-72,4)$ & 0,146 \\
\hline Ingesta calórica diaria (Kcal/día)* & $2052,4 \pm 262,8$ & $2231,3 \pm 254,6$ & $<0,001$ & $1689,4 \pm 173,6$ & $1820,1 \pm 176,1$ & $<0,001$ \\
\hline IMC* & $29,5 \pm 4,1$ & $27,3 \pm 4,2$ & $<0,001$ & $30,9 \pm 6,0$ & $27,0 \pm 5,4$ & $<0,001$ \\
\hline Obesidad (IMC =30)** & $44,5(38,6-50,4)$ & $25,1(23,4-26,8)$ & $<0,001$ & $53,6(48,3-58,9)$ & $26,6(25,1-28,1)$ & $<0,001$ \\
\hline
\end{tabular}

* Media \pm DE.

** Proporción (IC95\%). 
Tabla 3

Razón de ventajas en las personas con DM2 respecto a las que no padecen la enfermedad [OR (IC95\%)], ajustadas por edad y clase social. Se presenta también el coeficiente de regresión $( \pm E E)$, ajustado por edad y clase social, para mostrar el cambio en la ingesta energética diaria cuando se padece DM2

\begin{tabular}{|l|c|c|c|c|}
\hline & Hombres & $\mathbf{p}$ & Mujeres & p \\
\hline Tabaquismo* & $0,9(0,7-1,2)$ & 0,640 & $0,6(0,4-0,9)$ & 0,006 \\
\hline Sedentarismo* & $0,9(0,7-1,2)$ & 0,655 & $1,0(0,8-1,3)$ & 0,902 \\
\hline Obesidad (IMC = 30)* & $1,9(1,4-2,4)$ & $<0,001$ & $2,0(1,6-2,5)$ & $<0,001$ \\
\hline Ingesta calórica diaria (Kcal/día)** & $-37,3 \pm 12,9$ & 0,004 & $-56,8 \pm 9,4$ & $<0,001$ \\
\hline
\end{tabular}

* OR $\left(\mathrm{IC}_{95 \%}\right)$.

$* *(B \pm \mathrm{EE})$.

rencia significativa en el consumo de tabaco de los hombres diabéticos y no diabéticos y, además, los primeros mostraron mayor grado de sedentarismo $(p=0,022)$. En cambio las mujeres no presentaron diferencias para el sedentarismo pero sí para el tabaquismo puesto que las que padecían DM2 mostraban menor frecuencia de tabaquismo $(p<0,001)$. En ambos sexos, la ingesta energética declarada por las personas diabéticas fue menor y el IMC y la obesidad mayores (tabla2).

El 93,0\% $\left(\mathrm{IC}_{95 \%}=91,0-95,0\right)$ de la población con DM2 declaró un consumo de calorías procedentes de grasas saturadas superior al $10 \%$ de la ingesta energética y el $22,2 \%\left(\mathrm{IC}_{95 \%}=18,9-25,5\right)$ presentaba una concentración sérica de colesterol LDL menor de $100 \mathrm{mg} / \mathrm{dl}$. El 21,2\% ( $\mathrm{IC}_{95 \%}=17,9$ - 24,5) mostraba una presión sistólica menor de $130 / 80 \mathrm{mmHg}$, el 38,3\% $\left(\mathrm{IC}_{95 \%}=34,4\right.$ 42,2) tenía tratamiento antihipertensivo. La prevalencia de síndrome metabólico fue del $69,0 \%\left(\mathrm{IC}_{95 \%}=65,0-72,0\right)$.

El análisis multivariado permitió ajustar por edad y nivel socioeconómico para comprobar que no era menos probable que los varones fumaran o fueran sedentarios por ser diabéticos y que, aunque declararan menor ingesta calórica, era más probable que fueran obesos (tabla 3). Este mismo análisis mostró que las mujeres diabéticas sí presentaban mayor probabilidad de no fumar que las no diabéticas, pero eran igual de sedentarias y más obesas (tabla 3 ).

\section{DISCUSIÓN}

Según los resultados la población diabética de Canarias muestra hábitos de vida poco saludables. Su sedentarismo y obesidad son mayores que los existentes en la población general y sólo una pequeña proporción sigue una dieta baja en grasas saturadas. En los varones el hecho de saber que padecen DM2 ni siquiera se asocia a un abandono del tabaquismo.

Además, casi la mitad de los hombres y un tercio de las mujeres no siguen con regularidad un tratamiento para su DM2. La desventaja de los varones es atribuible a su mayor desconocimiento de la enfermedad y a la menor adherencia al tratamiento, lo cual puede estar en relación con la menor frecuentación de la atención primaria en el sexo masculino ${ }^{19}$. De hecho, las mujeres presentan unas cifras de DM2 no diagnosticada más bajas que en otros países ${ }^{20}$. Sólo los hombres se acercan al 25\% de DM2 no diagnosticada que presentan en EEUU las personas que disponen de aseguramiento médico ${ }^{21}$. En cualquier caso estas cifras, unidas al poco saludable estilo de vida, pueden explicar la mala evolución clínica de esta enfermedad en Canarias. Es posible que la mitad de los hombres con DM2 y la tercera parte de las mujeres sólo sigan adecuadamente su tratamiento cuando ya la enfermedad haya causado complicaciones. Los estudios previos llevados a cabo en el $\operatorname{archipiélago~}^{11,12}$ han medido frecuencia $\mathrm{y}$ calidad de tratamiento en pacientes que 
habían sido identificados de DM2 en atención primaria, quedando excluida la DM2 desconocida y aquélla que por razones atribuibles al paciente o al sistema sanitario no está incluida en programas de prevención secundaria y terciaria.

El presente estudio ha valorado la que hasta el momento es la mayor muestra de personas diabéticas seleccionadas de la población general de Canarias. No son pacientes seleccionados en consultas $\mathrm{o}$ registros sanitarios sino que se trata de una muestra aleatoria de la población de todo el archipiélago ${ }^{13}$, por lo que podemos considerar que la información obtenida representa el estilo de vida de las personas que padecen esta enfermedad en las islas. Cuando comparamos nuestros resultados con los obtenidos por procedimientos similares en poblaciones diabéticas en Norteamérica ${ }^{22}$, comprobamos que en el archipiélago los porcentajes de tratamiento son más bajos, aunque no alcancen la significación estadística, la presión arterial tiene peor control, el consumo reducido de grasas saturadas es menos frecuente y la proporción de individuos con concentración ideal de LDL colesterol es menor. La frecuencia de obesidad y el síndrome metabólico es parecida en ambas poblaciones pero los norteamericanos tienen en promedio 6 años más, por lo que en caso de que se pudiera estandarizar la edad los resultados serían aún más desfavorables para las personas diabéticas de Canarias.

En cuanto a España, no hemos encontrado un estudio previo de población diabética comparable al nuestro. Ante ello, hemos tomado como referencia el más amplio estudio efectuado en pacientes diabéticos de atención primaria ${ }^{23}$. Era, pues, de esperar que presentaran un porcentaje de tratamiento superior al de nuestro estudio que, como ya se dijo, no analiza pacientes sino población general. Esto, junto a los 10 años de edad en que dichos pacientes superan a nuestra población de estudio, ayuda a expli- car las grandes diferencias en la frecuencia de tratamiento antihipertensivo o hipolipemiante y en los valores de LDL colesterol y de presión arterial, siempre desfavorables en las islas. No obstante, el valor más llamativo de la población diabética del archipiélago vuelve a estar en la prevalencia de obesidad y síndrome metabólico que son, respectivamente, 15 y 19 puntos porcentuales mayor en Canarias que en la Península, pese a que la población de las islas es 10 años más joven. Esta prevalencia es consistente con otros estudios, donde ya se ha puesto de manifiesto la alta prevalencia de obesidad y síndrome metabólico en la población general de Canarias ${ }^{3,13,24}$.

Las actuales recomendaciones sobre el tratamiento de la DM2 y de la prevención cardiovascular en general insisten en la gran importancia que tiene el adecuado cumplimiento de las medidas relacionadas con el abandono del tabaquismo ${ }^{25}$, una dieta con alimentos y calorías adaptadas a las características de cada paciente ${ }^{26}$ y una correcta cantidad y calidad de actividad física $^{27}$. Son numerosos los estudios que han demostrado que una aplicación adecuada de estas medidas pueden reducir o retrasar la aparición de DM2, así como mejorar el grado de control de los pacientes que ya la padecen ${ }^{28-32}$. Por tanto, con el nivel de evidencia científica actual, cualquier sistema sanitario debe actuar intensamente sobre tabaquismo, sedentarismo y obesidad en la diabetes, y en Canarias esto resulta urgente.

Uno de nuestros resultados más preocupantes es el porcentaje de personas que sabiendo que padecen DM2 declaran no seguir tratamiento regularmente. La cuarta parte de los varones y la quinta de las mujeres que conocen su DM2 no están en tratamiento o no lo cumplen diariamente. Nuevos estudios que corroboren estos resultados deberían indagar si estamos ante un caso de menosprecio cultural de esta enfermedad por parte de una población que 
puede estar identificando la DM2 como un problema menor con el cual mucha gente vive una larga vida. Resultados similares a éstos se han encontrado en EEUU, con un cuarto de la población con DM2 mostrando pobre adherencia al tratamiento farmacológico en función de sus creencias sobre la enfermedad y los efectos secundarios de los medicamentos ${ }^{33}$. Aunque la aplicación de guías de práctica clínica depende tanto de la estructura del sistema sanitario como de la motivación y la formación de los profesionales sanitarios, no es menos cierto que las actitudes de los pacientes pueden ser determinantes en este empeño ${ }^{34}$. La inercia clínica es un factor bien conocido ${ }^{35}$, pero las barreras culturales, psicológicas y sociales dependientes de los pacientes están igualmente documentadas en la DM2 desde edades juveniles ${ }^{36}$ hasta edades adultas y avanzadas $^{37,38}$.

Una limitación de nuestro trabajo radica en que no se diseñó específicamente para estudiar el cumplimiento terapéutico sino que es un corte transversal de una cohorte de población general pero, paradójicamente, en ello se funda también parte de su interés, pues aporta una visión del conjunto de las personas diabéticas tal como viven en la comunidad, sin que su actitud esté mediatizada por su condición de pacientes.

Concluimos que la población con DM2 en Canarias, que es la de mayor mortalidad por esta causa y mayor morbimortalidad cardiovascular en España, muestra un estilo de vida poco saludable, con alto consumo de grasas saturadas y exposición al sedentarismo y la obesidad, y una elevada prevalencia de síndrome metabólico. Esto más el bajo porcentaje de personas con DM2 que siguen tratamiento con regularidad, puede explicar la mala evolución clínica de la enfermedad en esta Comunidad. El problema es más severo en los varones con DM2, que además mantienen el tabaquismo. Es necesario aumentar el esfuerzo realizado hasta la fecha para identificar a estas perso- nas, ofrecerles los programas preventivos de DM2 en atención primaria, educarlas sobre los cuidados de su enfermedad y ayudarles a mejorar su estilo de vida.

\section{BIBLIOGRAFÍA}

1. Centro Nacional de Epidemiología. Mortalidad por Causa, Sexo y Comunidad Autónoma. Tasas ajustadas por edad. [última consulta, 11 de junio de 2009]. Disponible en: http://www.isciii.es/htdocs/centros/epidemiologia/anexos/ww9201_cau_ca_tasae.htm.

2. Alfaro Latorre M, Regidor Poyatos E, Gutiérrez Fisac JL, Mataix González R, Guevara García D. Mortalidad por cáncer, por enfermedad isquémica del corazón, por enfermedades cerebrovasculares y por diabetes mellitus en España. Madrid: Instituto de Información Sanitaria, Ministerio de Sanidad; 2008.p. 20-26 y 39. [citado el 18 de mayo de 2009]. Disponible en: http://www.msc.es/estadEstudios/estadisticas/docs/MORTALIDAD _POR_ENFERMEDADES_CRONICAS.pdf

3. Cabrera de León A, Rodríguez Pérez MC, del Castillo Rodríguez JC, Brito Díaz B, Pérez Méndez L1, Muros de Fuentes M, et al. Estimación del riesgo coronario en la población de Canarias aplicando la ecuación de Framingham. Med Clin (Barc). 2006; 126: 521-526.

4. Arrieta Lezama J, Gutiérrez Ávila G, Moreno Alía I, Sierra Yébenes T, Estébanez C, Olmos Linares AM, et al. Informe de Situación de Diálisis y Trasplante en España, 2005. Nefrología. 2008; 28: 151-158.

5. Lorenzo V, Sanchez E, Vega N, Hernandez D. Renal replacement therapy in the Canary Islands: demographic and survival analysis. J Nephrol. 2006; 19: 97-103.

6. Boix R, Cañellas S, Almazán J, Cerrato E, Meseguer M, Medrano MJ. Mortalidad cardiovascular en España. Año 2000. Bol Epidemiol Semanal. 2003; 11: 241-244.

7. Maca-Meyer N, Villar J, Perez-Mendez L, Cabrera A, Flores C. A tale of aborigins, conquerors and slaves: Alu insertion polymorphisms and the peopling of Canary Islands. Ann Hum Genet. 2004; 68: 600-605.

8. García Quesada A. Cultura, antropología y etnografía en Canarias [última consulta, 19 de mayo de 2009]. Disponible en: http://www.gobiernode- 
canarias.org/educacion/ culturacanaria/ ant rop/antropo.htm.

9. de Pablos-Velasco PL, Martínez-Martín FJ, Rodríguez-Pérez F, Anía BJ, Losada A, Betancor P. Prevalence and determinants of diabetes mellitus and glucose intolerance in a Canarian Caucasian population - comparison of the 1997 ADA and the 1985 WHO criteria. Guía Study. Diabet Med. 2001; 18: 235-241.

10. Boronat M, Varillas VF, Saavedra P, Suárez V, Bosch E, Carrillo A, Nóvoa FJ. Diabetes mellitus and impaired glucose regulation in the Canary Islands (Spain): prevalence and associated factors in the adult population of Teide, Gran Canaria. Diabet Med. 2006; 23: 148-155.

11. de Pablos-Velasco PL, Martínez-Martín FJ, Molero R, Rodríguez-Pérez F, García-Puente I, Caballero A. Patterns of prescription of hypoglycaemic drugs in Gran Canaria (Canary Islands, Spain) and estimation of the prevalence of diabetes mellitus. Dab Metab. 2005; 3: 457-462.

12. Mora-Fernández C, Muros M, Jarque A, GonzálezCabrera F, García-Pérez J, Navarro J. Características de los pacientes diabéticos referidos por primera vez a las consultas de atención especializada de Nefrología. Nefrología. 2007; 27: 154-161.

13. Cabrera de León A, Rodríguez Pérez MC, Almeida González D, Domínguez Coello S, Aguirre Jaime A, Brito Díaz B et al. Presentación de la cohorte "CDC de Canarias": objetivos, diseño y resultados preliminares. Rev Esp Salud Pública. 2008; 82: 519-534.

14. American Diabetes Association: clinical practice recommendations 2002. Expert Committee on the Diagnosis and Classification of Diabetes Mellitus. Diabetes Care. 2002; 25 (Supl1): S1-147.

15. Cabrera de León A, Rodríguez Pérez MC, Rodríguez Benjumeda LM, Anía Lafuente B, Brito Díaz B, Muros de Fuentes M et al. Sedentarismo: tiempo de ocio activo frente al porcentaje del gasto energético. Rev Esp Cardiol. 2007; 69: 244-250.

16. National Cholesterol Education Program. Third Report of the National Cholesterol Education Program (NCEP) Expert Panel on Detection, Evaluation, and Treatment of High Blood Cholesterol in Adults (Adult Treatment Panel III) final report. Circulation. 2002; 106: 3188-3190.

17. A Cabrera de León, MC Rodríguez Pérez, S Domínguez Coello, C Rodríguez Díaz, C Rodríguez Álvarez, A Aguirre Jaime y el grupo CDC. Validación del modelo REI para medir la clase social en población adulta. Rev Esp Salud Pública. $2009 ; 83$ : 231-242.

18. A Aguirre Jaime, A. Cabrera de León, S Domínguez Coello, C Borges Álamo, L Carrillo Fernández, JC Gavilán Batista, MC Rodríguez Pérez, y D Almeida González. Validación de un cuestionario de frecuencia de consumo de alimentos adaptado para el estudio y seguimiento de la población de las Islas Canarias. Rev Esp Salud Pública. 2008; 82: 509-518.

19. Vedsted P, Christensen MB. Frequent attenders in general practice care: a literature review with special reference to methodological considerations. Public Health. 2005; 119: 118-137.

20. Norris SL, Kansagara D, Bougatsos C, Rongwel F. Screeening Adults for Type 2 Diabetes: A Review of the Evidence for the U.S. Preventive Services Task Force. Ann Intern Med. 2008; 148: 855-868.

21. Zhang X, Geiss LS, Cheng YJ, Beckles GL, Gregg EW, Kahn HS. The missed patient with diabetes: how access to health care affects the detection of diabetes. Diabetes Care. 2008; 31: 1748-1753.

22. Resnick HE, Foster GL, Bardsley J, Ratner RE. Achievement of American Diabetes Association clinical practice recommendations among U.S. adults with diabetes, 1999-2002: the National Health and Nutrition Examination Survey. Diabetes Care. 2006; 29: 531-537.

23. Orozco-Beltrán D, Gil-Guillen VF, Quirce F, Navarro-Pérez J, Pineda M, Gómez-de-la-Cámara A et al. Collaborative Diabetes Study Investigators. Control of diabetes and cardiovascular risk factors in patients with type 2 diabetes in primary care. The gap between guidelines and reality in Spain. Int J Clin Pract. 2007; 61: 909-915.

24. Álvarez León EE, Ribas Barba L, Serra Majem Ll. Prevalencia del síndrome metabólico en la población de la Comunidad Canaria. Med Clin (Barc). 2003; 120: 172-174.

25. American Diabetes Association. Smoking and diabetes (Position Statement). Diabetes Care. 2004; 27 (Suppl 1): S74-75.

26. American Diabetes Association. Nutrition Recommendations and Interventions for Diabetes. Diabetes Care. 2008; 31 (Suppl 1): S61-78.

27. Sigal RJ, Kenny GP, Wasserman DH, CastanedaSceppa C, White RD. Physical activity/exercise and type 2 diabetes: a consensus statement from the American Diabetes Association. Diabetes Care. 2006; 29: 1433-1438. 
28. Yusuf S, Hawken S, Ounpuu S, Dans T, Avezum A, Lanas F, McQueen M, Budaj A, Pais P, Varigos J, Lisheng L. Effect of potentially modifiable risk factors associated with myocardial infarction in 52 countries (the INTERHEART study): case -control study. Lancet. 2004; 364: 937-952.

29. Ramachandran A, Snehalatha C, Mary S, Mukesh B, Bhaskar AD, Vijay V: The Indian Diabetes Prevention Programme shows that lifestyle modification and metformin prevent type 2 diabetes in Asian Indian subjects with impaired glucose tolerance (IDPP-1). Diabetologia. 2006; 49: 289-297.

30. Pastors JG, Franz MJ, Warshaw H, Daly A, Arnold MS: How effective is medical nutrition therapy in diabetes care? J Am Diet Assoc. 2003; 103: 827831.

31. Orozco LJ, Buchleitner AM, Giménez-Perez G, Roqué I Figuls M, Richter B, Mauricio D. Exercise or exercise and diet for preventing type 2 diabetes mellitus. Cochrane Database Syst Rev. 2008 ; 3: CD003054.

32. Boule' NG, Kenny GP, Haddad E, Wells GA, Sigal RJ: Meta-analysis of the effect of structured exercise training on cardiorespiratory fitness in type 2 diabetes mellitus. Diabetologia. 2003; 46: 1071-1081.

33. Mann DM, Ponieman D, Leventhal H, Halm EA. Predictors of adherence to diabetes medications: the role of disease and medication beliefs. J Behav Med. 2009; 32: 278-284.

34. Cabana M, Rand C, Powe N, Wu A, Wilson M, Abboud PA, Rubin H. Why Don't Physicians Follow Clinical Practice Guidelines? A Framework for Improvement. JAMA. 1999; 282: 14581465 .

35. Shah BR, Hux JE, Laupacis A, Zinman B, van Walraven C. Clinical inertia in response to inadequate glycemic control: do specialists differ from primary care physicians? Diabetes Care. 2005; 28 : 600-606.

36. Rothman RL, Mulvaney S, Elasy TA, Vander Woude A, Gebretsadik T, Shintani A, Potter A, Russell WE, Schlundt D. Self-management behaviors, racial disparities, and glycemic control among adolescents with type 2 diabetes. Pediatrics. 2008; 121: e912-919.

37. Peyrot M, Rubin RR, Lauritzen T, Snoek FJ, Matthews DR, Skovlund SE. Psychosocial problems and barriers to improved diabetes management: results of the Cross-National Diabetes Attitudes, Wishes and Needs (DAWN) Study. Diabet Med. 2005; 22: 1379-1385.

38. Gonsalves WC, Gessey ME, Mainous AG 3rd, Tilley BC. A study of lower extremity amputation rates in older diabetic South Carolinians. J S C Med Assoc. 2007; 103: 4-7. 